\title{
Genetic characterization of nodular worm infections in Asian Apes
}

\author{
Erhan Yalcindag ${ }^{1,2}$, Peter Stuart ${ }^{1,3}$, Hideo Hasegawa ${ }^{4}$, Adrian Streit $^{5}$, Jana Doležalová 6 , \\ Helen Morrogh-Bernard ${ }^{7,8}$, Susan M. Cheyne ${ }^{8}$, Wisnu Nurcahyo ${ }^{9}$ \& Ivona Foitová1 ${ }^{\circledR}$
}

Parasitic nematodes of Oesophagostomum spp., commonly known, as 'nodular worms' are emerging as the most widely distributed and prevalent zoonotic nematodes. Oesophagostomum infections are well documented in African non-human primates; however, the taxonomy, distribution and transmission of Oesophagostomum in Asian non-human primates are not adequately studied. To better understand which Oesophagostomum species infect Asian non-human primates and determine their phylogeny we analysed 55 faecal samples from 50 orangutan and 5 gibbon individuals from Borneo and Sumatra. Both microscopy and molecular results revealed that semi-wild animals had higher Oesophagostomum infection prevalence than free ranging animals. Based on sequence genotyping analysis targeting the Internal transcribed spacer 2 of rDNA, we report for the first time the presence of $O$. aculeatum in Sumatran apes. Population genetic analysis shows that there is significant genetic differentiation between Bornean and Sumatran $O$. aculeatum populations. Our results clearly reveal that $O$. aculeatum in free-ranging animals have a higher genetic variation than those in semi-wild animals, demonstrating that $O$. aculeatum is circulating naturally in wildlife and zoonotic transmission is possible. Further studies should be conducted to better understand the epidemiology and dynamics of Oesophagostomum transmission between humans, non-human primates and other wild species and livestock in Southeast Asia.

Orangutans and gibbons are the only ape species living in Southeast Asia and both are primarily arboreal species. Orangutans show a $97 \%$ genetic similarity with humans ${ }^{1}$. Today, orangutans are divided into three species: the Bornean orangutans (Pongo pygmaeus), the Sumatran orangutans (Pongo abelii) and Pongo tapanuliensis, which has recently been separated from $P$. abelii ${ }^{2}$. Gibbons are smaller apes belonging to the family Hylobatidae with four recognized genera: Hoolock, Nomascus, Hylobates and Symphalangus ${ }^{3}$. Overall, 20 gibbon species have been identified, and only Hylobates are reported in both Sumatra and Borneo whilst Symphalangus are only found in Sumatra and South Malaysia ${ }^{4}$. The Asian apes are currently listed by the IUCN as Critically Endangered ${ }^{5}$. These species are severely threatened by poaching, habitat conversion, destruction through deforestation and other human activities. These threats may increase the opportunities for contact with other wildlife species, livestock and humans resulting in increased disease transmission between them. Infectious diseases, possibly linked to environmental changes, may play a role in population declines through increased mortality and morbidity of these species ${ }^{6}$. In addition, the zoonotic potential of pathogens in non-human primates (hereafter referred to as NHPs) has received considerable attention due to increased contact between domestic communities and local primate species ${ }^{7}$. While several parasitic and viral infections are well documented in African apes, the distribution and transmission of parasites of Asian apes is still relatively neglected and or poorly understood ${ }^{8}$. However, in the last decades, new pathogens have been discovered in Asian apes ${ }^{9-13}$ as well as new studies of previously known ones $^{6,8,14-18}$. The health of the NHPs are affected by a large variety of soil-transmitted helminths (STHs), some with zoonotic potential, e.g. roundworms (Ascaris lumbricoides), whipworms (Trichuris trichiura), hookworms (Necator americanus, Ancylostoma duodenale), threadworms (Strongyloides stercoralis) and also nodular worms

\footnotetext{
${ }^{1}$ Department of Botany and Zoology, Faculty of Science, Masaryk University, Kotlářská 2, 61137 Brno, Czech Republic. ${ }^{2}$ The Roslin Institute, Royal (Dick) School of Veterinary Studies, University of Edinburgh, Easter Bush, Midlothian EH25 9RG, UK. ${ }^{3}$ Department of Biological and Pharmaceutical Sciences, Munster Technological University, Tralee, Co. Kerry, Ireland. ${ }^{4}$ Department of Biomedicine, Faculty of Medicine, Oita University, 1-1 Idaigaoka, Hasama, Yufu, Oita 879-5593, Japan. ${ }^{5}$ Department Evolutionary Biology, Max Planck Institute for Developmental Biology, Max-Planck-Ring 9, 72076 Tübingen, Germany. ${ }^{6}$ Department of Physiology, Faculty of Veterinary Medicine, University of Veterinary and Pharmaceutical Sciences, Brno, Palackého tř. 1, Brno, Czech Republic. ${ }^{7}$ Borneo Nature Foundation, Palangkaraya, Central Kalimantan, Indonesia. ${ }^{8}$ Department of Humanities and Social Sciences, Oxford Brookes University, Oxford, UK. ${ }^{9}$ Department of Parasitology, Faculty of Veterinary Medicine, Gadjah Mada University, Yogyakarta, Indonesia. ${ }^{\circledR}$ email: ivona_foi@hotmail.com
} 


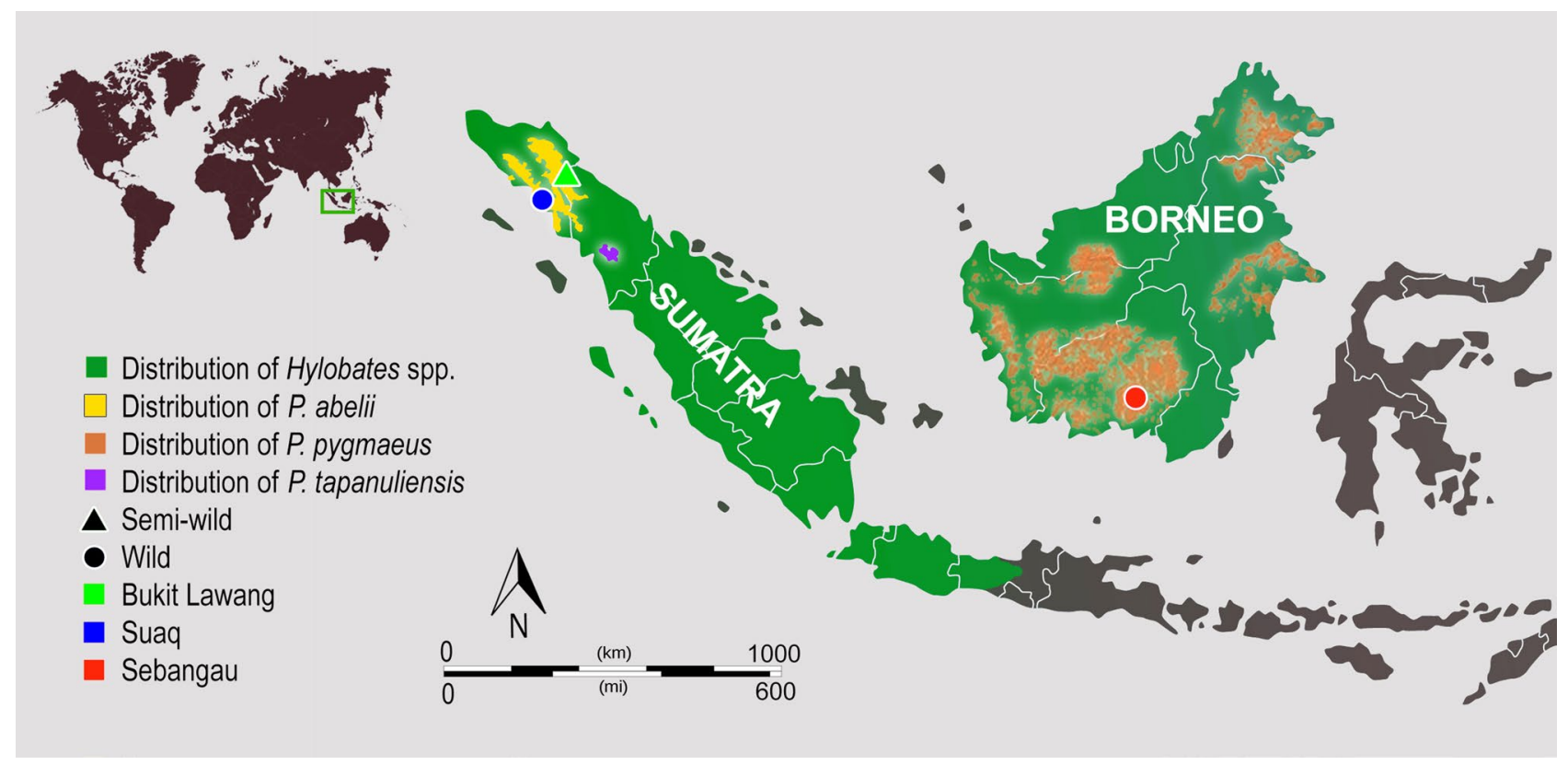

Figure 1. Geographical location of study sites in Borneo and Sumatra. For more information, please refer to Table 1 and Supporting Table S1. The map background was downloaded from free maps platform system (http:// www.freepik.com) and modified in Adobe Photoshop CS6.

(Oesophagostomum spp. $)^{19-25}$. Increased contact with humans has been identified as a risk factor of infection, making primates who have undergone human rehabilitation (semi wild) more likely to be infected than wild individuals ${ }^{12}$.

Oesophagostomum species are stout, white roundworms and have a direct life cycle. Eggs passed in the faeces, hatch and rapidly develop into L1 rhabditiform larvae. After $24 \mathrm{~h}$ of hatching, L2 become infective L3 within 3-4 days. L3 keep the protective cuticle of L2 and are capable of surviving long periods of adverse environmental conditions, e.g., hot-dry conditions of the dry season. Infection occurs by ingestion of filariform L3 larvae via vegetation eaten by the host. After ingestion, L3 pass to the cecum, where they exsheath within $~ 3$ days of ingestion then invade the tunica mucosa, stimulating the formation of separate cysts around individual larvae in the gut wall. The larvae develop there to the L4 stage then once in the lumen, the larvae moult and reach the adult stage ${ }^{26}$.

For most nematodes parasites, species identification based on egg morphology is difficult, therefore recently molecular techniques are widely used to identify species. Accordingly, recent molecular genetic studies have demonstrated that the ITS nucleotide sequence of rDNA allows an unequivocal identification and discrimination of a range of strongylid nematode species, irrespective of the developmental stage of the parasites ${ }^{27}$.

Based on molecular findings, Oesophagostomum spp. are known to frequently infect domestic and wild pigs, ruminants and primates ${ }^{28-31}$. Eight species of Oesophagostomum have been observed to occur in African NHPs and three of them (O. bifurcum, O. stephanostomum and O. aculeatum) are also reported in humans ${ }^{32-34}$. Human cases have been attributed to an animal origin and NHPs have been proposed as a potential reservoir ${ }^{33}$. Infections in wild primates appear to be asymptomatic, although clinical signs and mortality due to Oesophagostomum have been recorded in captive setting $s^{35}$. Oesphagostomum spp. have been reported from orangutans based on morphological and coprological analysis ${ }^{36}$. Arizono et al. ${ }^{37}$ molecularly identified O. aculeatum infections for the first time in Japanese macaques. Recently, molecular markers showed a widespread distribution of O. aculeatum in the Bornean primate community, including orangutan ${ }^{38}$, although there is a paucity of investigations in gibbons. Currently, there is no study done on O. aculeatum distribution in the Sumatran NHPs community. The aim of this study was therefore to genetically investigate Oesphagostomum parasitising in wild and semi-wild Asian apes (two orangutan species; P. pygmaeus and P. abelii and one gibbon species; Hylobates albibarbis) to confirm for the first time if Sumatran orangutans and wild Asian gibbons are being infected and to investigate the distribution and population structure of nodular worm infections in these species.

\section{Results}

A total of 55 faecal samples from 50 different orangutans and 5 from different gibbon individuals were collected from three different site. (Fig. 1, Table 1 and Supp Table 1). From these, 35 orangutans and 3 gibbons appeared to have Oesophagostomum and/or hookworm nematode-like eggs by microscopy, population-wide prevalence of infection 69\% (ranging 52.6\%-90\% (Table 1). All eggs identified by microscopy as Oesophagostomum were similar in internal and external morphology in samples from all primate species. The size of eggs was $60-85$ by $35-50 \mu \mathrm{m}$, similar to previous records ${ }^{28,39}$. For each population, the mean size of the eggs measured was calculated and no significant difference between populations was observed (chi-square $=0.063, \mathrm{df}=2, \mathrm{p}$-value $>0$. 05) (Supp Table 3). 


\begin{tabular}{|c|c|c|c|c|c|c|c|c|c|c|c|}
\hline Site & Host species & Population & Short Name & Forest type & Density & \begin{tabular}{|l} 
Latitude \\
Longitude
\end{tabular} & Sample size & $\begin{array}{l}\text { Positive } \\
\text { samples }\end{array}$ & $\begin{array}{l}\text { Prevalence } \\
(\% 95)\end{array}$ & Males (n) & $\begin{array}{l}\text { Females } \\
(\mathbf{n})\end{array}$ \\
\hline Borneo $^{\dagger}$ & $\begin{array}{l}\text { Pongo pyg- } \\
\text { maeus }\end{array}$ & Sebangau & Seb & Peat swamp & $\begin{array}{l}2.3 \mathrm{ind} / \\
\mathrm{km}^{273}\end{array}$ & $\begin{array}{l}2^{\circ} 07^{\prime} \mathrm{S} \\
112^{\circ} 22^{\prime} \mathrm{E}\end{array}$ & 11 & $6^{\mathrm{a}} / 3^{\mathrm{b}}$ & $\begin{array}{l}54.55^{\mathrm{a}}(28- \\
79) 27.27^{\mathrm{b}} \\
(10-57)\end{array}$ & $2^{\mathrm{a}}\left(7^{\mathrm{a}}\right)$ & $1^{\mathrm{a}}\left(4^{\mathrm{a}}\right)$ \\
\hline Borneo $^{\dagger}$ & $\begin{array}{l}\text { Hylobates } \\
\text { albibarbis }\end{array}$ & & & & $\begin{array}{l}2.59 \text { groups/ } \\
\mathrm{km}^{2} 74\end{array}$ & & 5 & $3^{\mathrm{a}} / 3^{\mathrm{b}}$ & $\begin{array}{l}60^{\mathrm{a}}(23-88) \\
60^{\mathrm{b}}(23-88)\end{array}$ & $\varsigma$ & \\
\hline Sumatra $^{\dagger}$ & Pongo abelii & Suaq & Suaq & $\begin{array}{l}\text { Coastal } \\
\text { swamp }\end{array}$ & $7 \mathrm{ind} / \mathrm{km}^{2} 75$ & $\begin{array}{l}3^{\circ} 42^{\prime} \mathrm{N} \\
97^{\circ} 26^{\prime} \mathrm{E}\end{array}$ & 19 & $10^{\mathrm{a}} / 6^{\mathrm{b}}$ & $\begin{array}{l}52.63^{\mathrm{a}}(32- \\
73) 31.58^{\mathrm{b}} \\
(15-54)\end{array}$ & $4^{\mathrm{a}}\left(13^{\mathrm{a}}\right)$ & $2^{\mathrm{a}}\left(6^{\mathrm{a}}\right)$ \\
\hline Sumatra & Pongo abelii & Bukit Lawang & Buk & $\begin{array}{l}\text { Hill diptero- } \\
\text { carp }\end{array}$ & $\begin{array}{l}1.8 \mathrm{ind} / \\
\mathrm{km}^{2} 73\end{array}$ & $\begin{array}{l}3^{\circ} 47^{\prime} \mathrm{N} \\
98^{\circ} 08^{\prime} \mathrm{E}\end{array}$ & 20 & $18^{\mathrm{a}} / 15^{\mathrm{b}}$ & $\begin{array}{l}90^{\mathrm{a}}(70-97) \\
75^{\mathrm{b}}(53-89)\end{array}$ & $5^{\mathrm{a}}\left(7^{\mathrm{a}}\right)$ & $10^{\mathrm{a}}\left(13^{\mathrm{a}}\right)$ \\
\hline Total & & & & & & & 55 & $37^{\mathrm{a}} / 27^{\mathrm{b}}$ & $\begin{array}{l}69.1^{\mathrm{a}}(56-80) \\
49.1^{\mathrm{b}}(36-62)\end{array}$ & $11^{\mathrm{a}}\left(27^{\mathrm{a}}\right)^{\mathrm{x}}$ & $13^{\mathrm{a}}\left(23^{\mathrm{a}}\right)^{\mathrm{y}}$ \\
\hline
\end{tabular}

Table 1. Number of Asian ape's fecal samples collected and sequenced in this study with site, populations, forest type, geographical coordinates, total sample size, and number of infected samples. ${ }^{\text {a Positive samples }}$ from microscopy. ${ }^{b}$ Positive samples from PCR. ${ }^{\dagger}$ Populations of free ranging animals. ${ }^{\ddagger}$ Populations of semiwild animals. ${ }^{\S} 3$ of 6 gibbons sex were unknown, data not include in the table. ${ }^{\ddagger}$ Infected orangutan samples by sex.

Polymerase Chain Reaction (here after, PCR) generated single clear amplicons from both the primer couples, of expected length for all microscopy-positive individuals. Despite PCR positive Oesophagostomum infection, with a prevalence of $49.1 \%$ [95\% CI; 36.3\%-61.9\%], the microscopy prevalence was $69.1 \%$ [ $95 \%$ CI; $55.9 \%-79.7 \%$ ] (Table 1). 11 positive samples from microscopy were negative by PCR.

Based on PCR positive results, no significant difference was found in the frequency of infection between the wild populations of the two different species ( $P$. abelii $31.6 \%, \mathrm{~N}=19$, P. pygmaeus $27.3 \%, \mathrm{~N}=11 ; \chi 2=0.062,1$ df; $\mathrm{p}=0.8041$ ), as well as between all Sumatran and Bornean orangutan samples (all P. abelii 53.8\%, $\mathrm{N}=39, P$. pygmaeus; $27.8 \%, \mathrm{~N}=11 ; \chi 2=1.479,1 \mathrm{df} ; \mathrm{p}=0.2239$ ). Semi-wild orangutans had significantly higher frequencies of infection with $O$. aculeatum than wild orangutans (semi-wild $75 \%, \mathrm{~N}=20$, wild $30 \%, \mathrm{~N}=30 ; \chi 2=8.016,1 \mathrm{df}$; $\mathrm{p}=0.0046$ ). The same patterns observed between only $P$. abelii semi-wild and wild individuals (semi-wild $P$. abelii $75 \%, \mathrm{~N}=20$, wild $P$. abelii $31.57 \%, \mathrm{~N}=19 ; \chi 2=5.748,1 \mathrm{df} ; \mathrm{p}=0.0165$ ). No significant association was detected between orangutan sex and frequency of infection with $O$. aculeatum (female 56.5\%, $\mathrm{N}=23$, male $40.7 \%, \mathrm{~N}=27$; $\chi 2=0.688,1 \mathrm{df} ; \mathrm{p}=0.407)$. Regarding the wild male and wild female individuals, no significant difference was found (wild male $30 \%, \mathrm{~N}=20$, wild female $30 \%, \mathrm{~N}=10 ; \chi^{2}=0.1 \mathrm{df} ; \mathrm{p}=1$ ). The same patterns was also observed in both semi-wild both female and male orangutans (semi-wild female $76.4 \%, \mathrm{~N}=13$, semi-wild male $71.4 \%$, $\left.\mathrm{N}=7 ; \chi^{2}=0.073,1 \mathrm{df} ; \mathrm{p}=0.7866\right)$.

However, we did not find any significant difference in the frequency of $O$. aculatum infection within the two sympatric different host species; (P. pygmaeus $27.3 \%, \mathrm{~N}=11, \mathrm{H}$. albibarbis $60 \%, \mathrm{~N}=5 ; \chi 2=0.485,1 \mathrm{df} ; \mathrm{p}=0.4862$ ) or between all orangutans population versus gibbons (all orangutans $48 \%, \mathrm{~N}=50$, gibbons $60 \%, \mathrm{~N}=5 ; \chi 2=0.002$, $1 \mathrm{df} ; \mathrm{p}=0.966)$.

We obtained a 758-bp-length fragment of ITS1-5.8S-ITS2 ribosomal DNA (here after, rDNA) from a total of 27 different individuals, 3 from gibbons and 24 from orangutans. By comparison with published sequences using NCBI BLAST, our samples showed a 98\% identity for O. stephanostomum (AB821014.1), 95\% O. detantum (AJ619979.1) and 94\% Oesophagostomum sp. MOS-2014 (AB908964.1). The resulting long DNA sequence overlapped with published sequences and contained no insertions or deletions, making alignment unequivocal. After analysis of the 27 Oesophagostomum sequences, we identified seven unique haplotypes. H1, H4, H2 and $\mathrm{H} 3$ were the most frequent haplotype detected in $48 \%, 14 \%, 11 \%$ and $11 \%$ of the infections, respectively. There is no shared haplotype between the two wild orangutan species P. pygmaeus; Bornean Sebangau and P. abelii; Sumatran Suaq (hereafter referred to as Seb and Suaq respectively). Only two haplotypes (H1 and H2) are shared between the Sumatran populations of Suaq and the semi-wild population in Bukit Lawang (hereafter referred to as the Buk). H3 is the only haplotype shared between wild P. pygmaeus and H. albibarbis in Sumatra and H5, $\mathrm{H} 6$ and $\mathrm{H} 7$ are unique haplotypes represented only in Seb (Fig. 2a). The nucleotide position of polymorphic sites are presented in Fig. 2 b.

The best sequence evolution of Oesophagostomum sequences obtained in this study with published other Oesophagostomum species sequences including outgroup, were HKY86 + G6 by JModelTest ${ }^{40}$. Published reference sequences were included to identify putative species and only 258 bp sequence were available. Based on phylogenetic analyses of the Oesophagostomum ITS2 rDNA (258 bp) sequences, the phylogenetic tree resolved these sequences into 8 clades according to host species (Fig. 3). Clade 1 composed of all O. asperum sequences in one group and a second distinct $O$. venulosum group that are infecting mainly bovidae species. Clade 2 is represented by two groups. The first contains both O. quadrispinulatum and the second contained all O. dentatum sequences. Clade 3 contained all O. colombianum samples as well as Oesophagostomum sp. only infecting small domestic ruminants. Clade 4 is divided into 2 groups with, one O. sikae isolates from deer and all O. ruminatum sequences isolated from cattle.

The Clade 5 contained Oesophagostomum sp. only infected human and African monkeys. 


\begin{tabular}{|ll|}
$\square$ & Bukit Lawang \\
$\square$ & Suaq \\
$\square$ & Sebangau \\
$\bigcirc$ & Mutated positions \\
K & Gibbons \\
& Orangutans \\
\hline
\end{tabular}

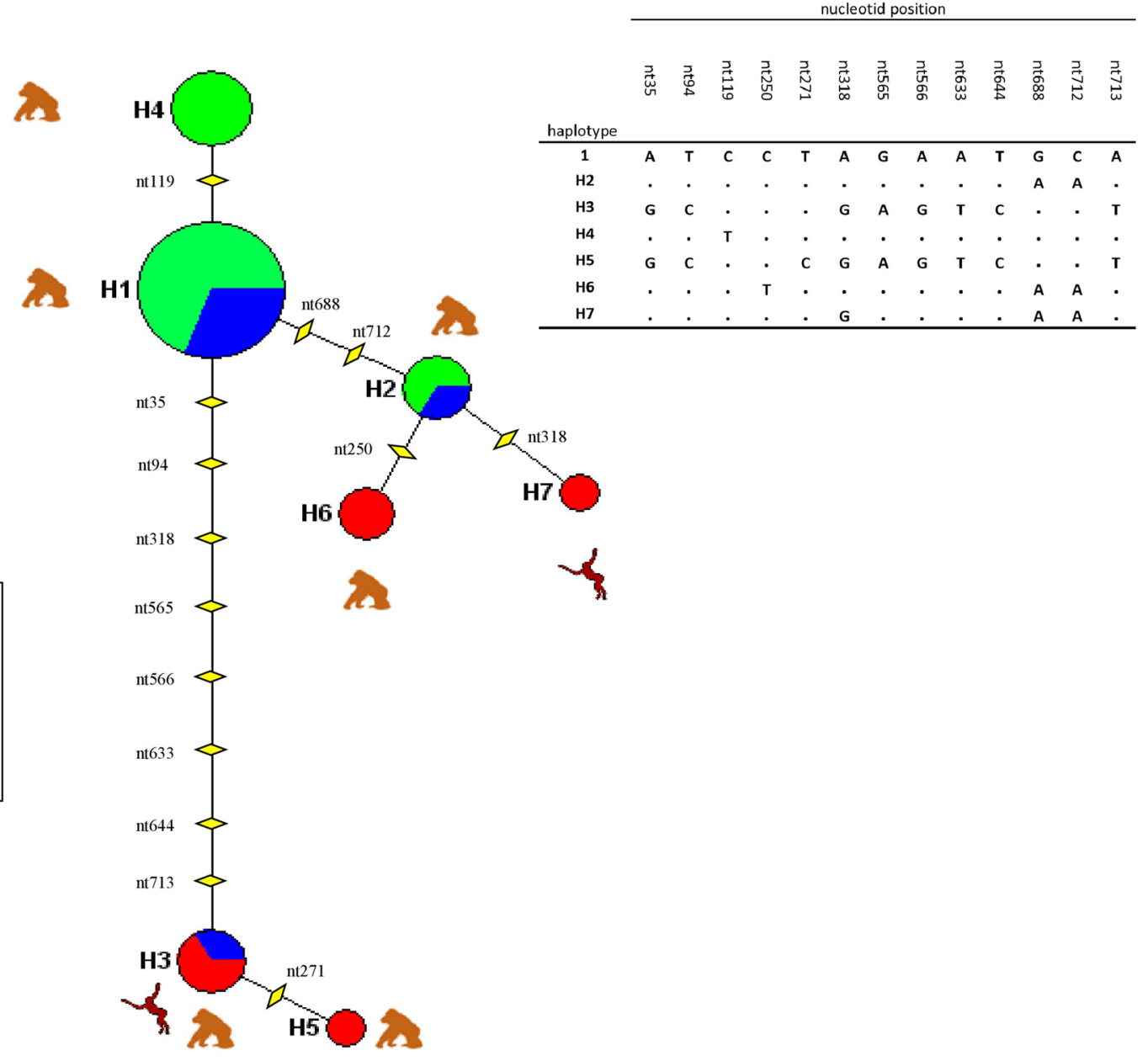

Figure 2. (a) Haplotype networks constructed using NETWORK 5.0.0.0. The network was built using a 758-bp-long fragment containing the ITS1-5.8S-ITS2 rDNA from 27 O. aculeatum specimen from this work. Node sizes (circles) are proportional to haplotype frequencies. Node colors indicate geographic origin. (b) Detected mutation positions on the 758-bp-long fragment containing the ITS1-5.8S-ITS2 rDNA.

The clade 7 contained all sequences of $O$. bifurcum and clade 8 contained all $O$. stephanostomum sequences from different host species. Clade 6 was composed of all orangutan sequences as well as three sequences from gibbons with all O. aculeatum sequences with a 96-99\% similarity.

Tree topologies showed a strong pattern of geographical structure across the geographic range of Oesophagostomum spp. between Southeast Asian and African primates. Our results show that Southeast Asian NHPs are infected by $O$. aculeatum only (Fig. 3). Within the clade 6, we observed two groups. The first group contained two sequences from gibbons and one sequence from Sumatran orangutan and one sequence from a Bornean orangutan. The second group contained all of the sequences from semi-captive Sumatran orangutans. Six additional sequences; one sequence from a gibbon, two sequences from two Bornean orangutans, two sequences from two semi-wild Sumatran orangutans and one sequence from one free ranging Sumatran orangutan sorted into group two which were $99 \%$ similar to the same O. aculeatum reference sequences.

The total number of sequences obtained and analysed for each population is given in Table 2. Overall, the Bornean (Seb) population exhibited the greatest haplotype diversity (0.867) and nucleotide diversity $(0.0089)$. However, the Sumatran populations showed almost the same haplotype diversity in both ( 0.6 for Suaq and 0.59 for Buk) but the Suaq population displayed four times higher nucleotide diversity than the Buk population (Table 2).

At host intra species level, nucleotide diversity estimated from the rDNA marker was 3.6- fold higher in Suaq (Sumatran wild) then Buk (Sumatran semi-wild). At host inter species level, Seb (Bornean wild) samples had the highest nucleotide diversity; 7.5 and 2 times higher than semi-wild and wild populations from Sumatra, respectively.

Genetic differentiation between Sumatran and Bornean populations was very strong as shown by high pairwise Fst estimates at rDNA (Table 3). Regarding genetic differentiation between populations, the lowest Fst values was observed between Buk and Suaq populations $(F s t=0.06882, P>0.05)$ while the highest significant genetic differentiation was observed between Buk and Seb $(F s t=0.49204, \mathrm{P}<0.05)$ (Table 3$)$. The analysis of the 

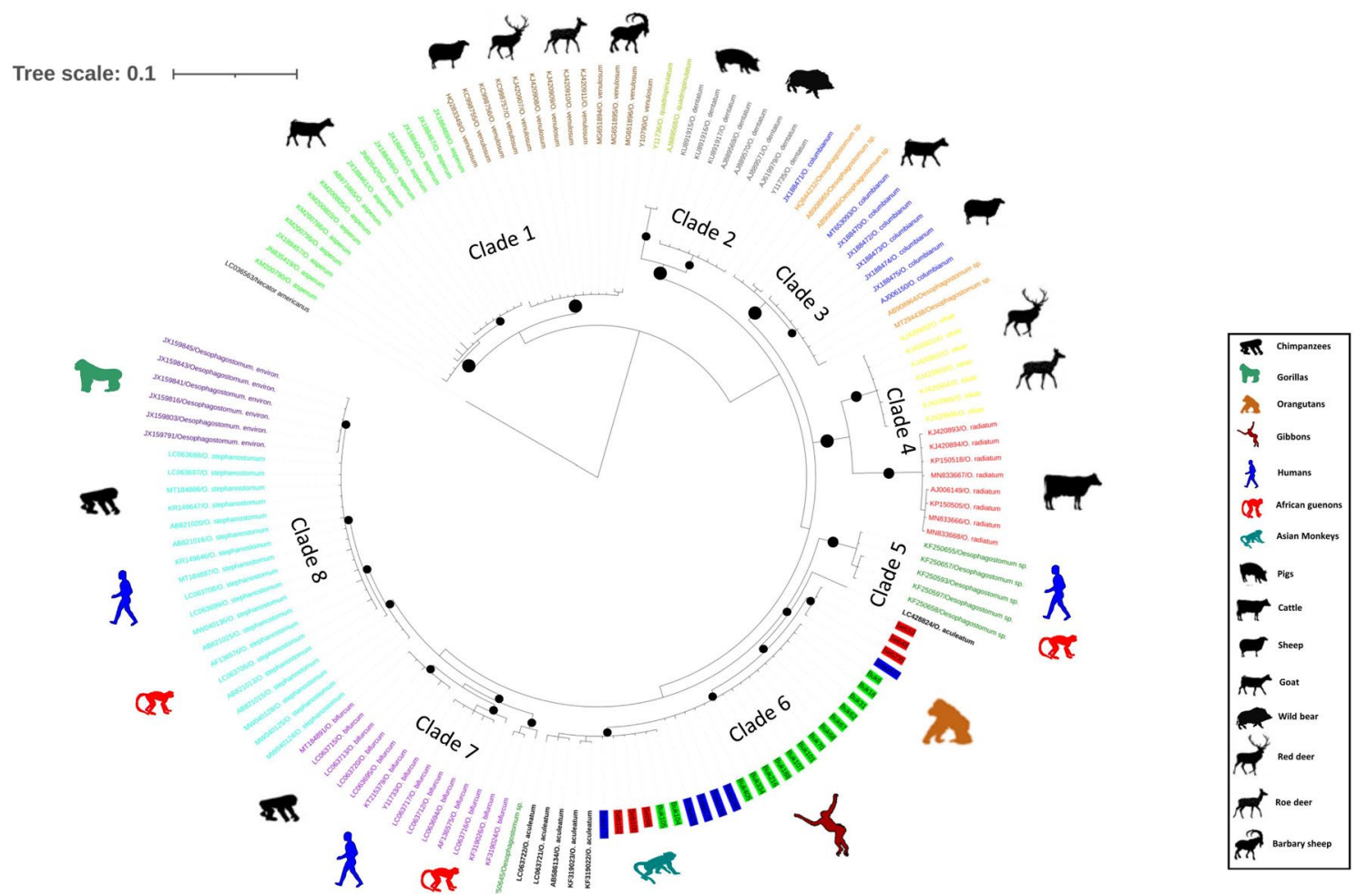

Figure 3. Phylogenetic relationship among Oesophagostomum isolates based on ITS2 rDNA (258 bp) sequences. The best-fitting model under the Akaike information criterion was HKY86 + G6 for nucleotides as identified by JModelTest v.0.1.1. Scale bar indicates nucleotide substitution per site. High (>80) bootstrap support are indicated next to the respective node by dots in black, respectively. O. aculeatum sequences are identified in this study are indicated with text-highlighted colour, sequences taken from NCBI are presented with font colour.

\begin{tabular}{|l|l|l|l|l|l|l|l|}
\hline Population & Size (bp) & N & S & h & Hd & $\pi$ & D \\
\hline Suaq & 758 & 6 & 10 & 3 & 0.6 & 0.0044 & 3.33 \\
\hline Buk & 758 & 15 & 3 & 3 & 0.59 & 0.0012 & 0.914 \\
\hline Seb & 758 & 6 & 12 & 4 & 0.867 & 0.0089 & 6.8 \\
\hline Total & 758 & 27 & 13 & 7 & 0.741 & 0.0044 & 3.345 \\
\hline
\end{tabular}

Table 2. Summary statistics on polymorphism of ITS1-5.8S-ITS2 genes in three populations of O. aculeatum collected from orangutans and gibbons inhabiting Borneo and Sumatra. N, Number of Sequences obtained; $S$, Number of polymorphic sites; h, Number of haplotype; Hd, Haplotype diversity; $\pi$, Nucleotide diversity; D, Average number of nucleotide difference between sequences.

\begin{tabular}{|l|l|l|l|}
\hline ITS1-5.8S-ITS2 & Suaq & Buk & Seb \\
\hline Suaq & & 0.18919 & 0.15315 \\
\hline Buk & $\mathbf{0 . 0 6 8 8 2}$ & & 0 \\
\hline Seb & $\mathbf{0 . 0 9 7 0 3}$ & $\mathbf{0 . 4 9 2 0 4}$ & \\
\hline
\end{tabular}

Table 3. Estimates of genetic differentiation $\left(\mathrm{F}_{\mathrm{ST}}\right)$ obtained between pairs of populations (in bold) and p-value of the test of differentiation (in italics) for ITS1-5.8S-ITS2 gene. 
three different $O$. aculeatum populations showed that there is no correlation between the genetic distances with geographic distance (Supplementary Figure S1).

\section{Discussion and conclusions}

In this study, we extracted DNA directly from nematodes eggs, without coprocultures and genetic characterization of L3 larvae, to identify nodular worm infection in Asian NHPs. Based on the internal transcribed spacer 2 (ITS 2) region of ribosomal RNA gene (rDNA), we confirmed that O. aculeatum is infecting Asian apes in Borneo. We confirm for the first time that Sumatran NHPs are also infected with this parasitic nematode. We confirm by molecular techniques that gibbons can be infected and therefore should be included in future studies of nodular worms, such as investigating how their unique ecology effects their risk of infection.

Mitochondrial and nuclear genes are used in phylogenetic studies on strongylid species ${ }^{41}$. Based on the mitochondrial cox 1 gene, Frias et al. ${ }^{38}$ showed that Bornean primates are subject to O. aculeatum infection. However, in our study we used ITS sequences to discriminate nematode species as the ITS regions are among the most variable nuclear loci, with a sufficient number of comparative sequences in databases. Our result demonstrated the same phylogenetic similarity observed from Frias et al $^{38}$. Accordingly, the rapid mode of ITS evolution including frequent indels makes alignment of ITS haplotypes more challenging compared to protein-coding markers, which can adversely affect the reliability of phylogenetic reconstruction ${ }^{42}$.

The evolution and dispersion of strongylid parasites and their relationships with other STHs species found in NHPs especially in hominids have been a point of attention ${ }^{28,36,43}$. Several studies have shown that Oesophagostomum infections of African primates are mainly O. stephanostomum, O. bifurcum and those belonging to the unclassified Oesophagostomum sp. and presented in three genetic lineages ${ }^{27,28,44,45}$. Later, Ota et al. ${ }^{46}$, showed the existence of a fourth clade, O. aculeatum clade from Asian primates, distinct from the three clades of African species. However, the molecular phylogenies have been limited by a deficiency of representative samples from Asian apes. In our study, based on ITS and 5.8S nucleotide sequence information, we identified Oesophagostomum sp. as one of the prevalent helminth parasites of Asian non-human primates. The Oesophagostomum sequences recovered from Asian apes clustered within the $O$. aculeatum clade. Interestingly, we did not detect any other nodular worm infections in our dataset, as it is known that the primer set used in our study are able to detect other Oesophagostomum species ${ }^{46}$, suggesting that $O$. aculeatum is the only or at least the predominant Oesophagostomum species that parasitizes orangutans and gibbons in our study area. Recently, Frias et al. ${ }^{38}$ showed that $O$. aculeatum is widely distributed in Borneo and that it infected all studied members of host species, such as long-tailed macaques, silvered langurs, proboscis monkeys and Bornean orangutans, with little clear genetic differentiation among them.

Previous studies have indicated that captive and/or semi-wild orangutans are subject to a higher prevalence of infection than wild ones ${ }^{12,47,48}$. Studies of terrestrial NHPs found a high STH prevalence of $81.2 \%$ in captive orangutans with ground dwelling behaviour ${ }^{8,47,49,50}$. In addition to these studies, our results significantly demonstrate that more semi-wild orangutans were positive for O. aculeatum than wild orangutans. However, it should be noted that this may be because of a sampling bias or over-representation of semi-wild orangutan samples in the dataset. As the presence of nodular worms have been confirmed in Asian primates in this and further studies with larger sample sizes should be carried out to confirm these findings.

However, several behavioural and ecological factors may contribute to higher infection with nodular worm species among semi-captive primates, such as restricted ranges making it difficult to avoid contaminated areas, changes in social structure, increased population density, dietary changes and stress and more contact with other animal host species. Another significant factor could be decreased arboreality and increased terrestrial locomotion in semi-captive animals, which allows more contact with the soil and increases exposure to STHs infective larvae. Regarding wild NHPs in Asia, previous studies have reported that the percentage of wild orangutans infected by STHs were $33 \%$ for P. pygmaeus ${ }^{8}$ and $47 \%$ for P. abelii ${ }^{47}$. In this study, we report that $30 \%$ of free ranging animals were infected with O. aculeatum alone. Apart from decreased arboreal life style and increased contact with soil, increasingly overlapping home ranges with other wildlife species and livestock may result in increased levels of infection and parasite occurrence ${ }^{8,47,51}$. Additionally, the habitat along the swamp forest seems to be an ideal environment for the development of soil-transmitted species as it provides the required humidity ${ }^{7}$.

In addition, Ghai et al. ${ }^{28}$ found that, in the case of Oesophagostomum, smaller primate groups with large daily travel distances had a higher prevalence. The free ranging orangutans have large home ranges, for example wild orangutans home ranges are estimated to be $423 \mathrm{ha} /$ month for males and $131 \mathrm{ha} /$ month for females $^{52}$. However, in our study the free ranging animals with larger habitat ranges were not observed to have higher prevalence's of infection than semi-wild animals. The highest prevalence of O. aculeatum in orangutans was found in Bukit Lawang (75\%) despite it having the lowest orangutan density $\left(1.8 \text { individuals } / \mathrm{km}^{2}\right)^{48}$, suggesting orangutan to orangutan transmission may not be a common source of infection directly or indirectly. Although Frias et al. ${ }^{38}$ suggested that orangutans may also acquire Oesophagostomum from other NHPs, in our study, this could be related to increased human activity in that area ${ }^{48}$ and increased approaching of humans, their dwellings and domestic animals or by semi-wild orangutans who have been previously exposed to human contact during rehabilitation. Ghai et al. ${ }^{28}$ observed that, some primates, particularly those traversing large distances in small groups, were most susceptible to nodule worm infection. If data were available to investigate the relationship between range size and prevalence in wild orangutans and gibbons, a similar pattern observed by Ghai et al. ${ }^{28}$ would be interesting to test.

The observed low infection prevalence from free ranging NHPs may also point towards the hypothesis that great apes are able to control parasite infection and avoid the progression of the disease by self-medication, i.e. leaf swallowing ${ }^{35,53,54}$ and wild animals have more access to these plants. Recently the first report of leaf 
swallowing in an Asian species, the white-handed gibbon, suggested a similar self-medicative function against nematode infection ${ }^{55}$.

In this study, the patterns of genetic variability observed among populations differ. A high level of genetic diversity was found across all three $O$. aculeatum populations. A significant difference in the number of polymorphic sites, nucleotide diversity and average number of nucleotide differences was evident between wild and semi-wild populations. Free ranging populations had higher genetic diversity than semi-wild ones. In wildlife, genetic diversity is probably greater than in anthropized environments, possibly due to a larger range of hosts ${ }^{56,57}$. Within free ranging populations, the Seb population had a higher genetic diversity then Suaq. This higher genetic diversity was due to observed unique haplotypes, geographical isolation, population size and different host species. Moreover, O. aculeatum, is a specialised organism that infects a specific host and the genetics and behaviour of a host can play an important role on the parasites genomic variation within the different host species (P. pygmaeus, P. abelii and H. albibarbis). Our assessment of population genetic differentiation calculating Fst index revealed non-significant differentiation among Sumatran populations and indicated low levels of genetic differentiation within the same host population. This pattern was also observed between the two free ranging populations, Seb and Suaq, from different host species. Interestingly our result showed significant genetic diversity between Buk and Seb populations. The isolation by distance analysis indicated that more genetically similar relationships were found for more distant populations then nearby populations (between Suaq and Seb then between two neighbour semi wild and wild Sumatran, Suaq and Buk population). Thus, O. aculeatum isolated from different geographical regions may differ because of a heterogeneous species, less population diffusion, complex genetic structure and high differentiation.

In our study, the molecular analysis was limited to a single gene. While several studies demonstrated a relatively high genetic diversity in the Oesophagostomum genus ${ }^{27,28,46}$, the sequencing of additional genes or other molecular markers should be applied to confirm species level differentiation and the population genetic structure of O. aculeatum in Southeast Asia.

The origin of O. aculeatum is unclear but it is clear that O. aculeatum is confined to Asia and infects more wild populations such as other primates and elephants $s^{37,38,58}$. Faecal examination of long-tailed macaques in Asia has uncovered Oesophagostomum spp. infections ${ }^{59}$. The Asian non-human primates including orangutans and gibbons, inhabiting the same area as elephants or other primates might provide evidence of O. aculeatum transmission between species from different environmental sources.

O. bifurcum and O. stephanostomum have been identified with certainty in reported Oesophagostomum human infections ${ }^{33}$, however, possible O. aculeatum infections have also been reported in humans in Southeast Asia ${ }^{60-62}$. To our knowledge, outbreaks of Oesophagostomosis in human populations have not been documented in Indonesia. Further work to test humans in the area and further genotyping would help to understand O. aculeatum infections in human.

Although other Oesophagostomum spp. such as O. stephanostomum and O. bifurcum are regarded as zoonotic ${ }^{33}$ the lack of studies characterising $O$. aculeatum in wildlife makes it different to understand the epidemiology of this species and further investigations are needed to tackle this problem. However, we do not reject the possibility of O. aculeatum having a zoonotic potential as already proposed by Frias et al..$^{38}$.

Our results confirm that $O$. aculeatum should be considered a pathogen for non-human primates in Southeast Asia. Oesophagostomum in semi-wild animals were found at a higher prevalence but showed a lower genetic variability compared with the ones in fully wild animals, further demonstrating the influence of human activities on the parasite dynamics in wild primate species. Further studies should be conducted to better understand the epidemiology and dynamic of Oesophagostomum transmission between human, non-human primates, other wild species and livestock, in Southeast Asia, following a one-health approach. Of particular interest are changes in transmission and prevalence in the context of the increasing human perturbation of natural forested habitats in Southeast Asia.

\section{Materials and methods}

Study site, sample collection and origin of faecal samples. Faecal samples were collected from three sites in Indonesia, one site in Borneo and two sites in Sumatra between 2004 and 2011. In Borneo, 11 faecal samples from orangutans (P. pygmaeus) and 5 samples from gibbons (H. albibarbis) were collected in Sebangau. Samples were collected within the LAHG (Laboratorium Alam Hutan Gambut: The Natural Laboratory for the Study of Peat Swamp Forest), an area of $500 \mathrm{~km}^{2}$ in the north-east of the Sebangau Forest, which was designated for the purpose of scientific research in 1997, and is managed by CIMTROP (Centre for International Cooperation in Management of Tropical Peatland) at the University of Palangka Raya. Sebangau is located in Central Kalimantan and consists of peat swamp forest. 39 faecal samples from Sumatran orangutans (P. abelii) were collected from two localities in the Gunung Leuser National Park. Suaq Balimbing is a coastal swamp situated on the western border of Gunung Leuser National Park. Bukit Lawang is located in Northern Sumatra, on the eastern border of the Gunung Leuser National Park, in hill dipterocarp forest (Fig. 1 and Table 1).

Except for orangutans in Bukit Lawang, all animals in this study can be considered wild. They have no physical contact with humans. In Bukit Lawang orangutans are considered semi-wild because they were released after a reintroduction process and therefore are less hesitant to have contact with humans ${ }^{12}$.

Wild and semi-wild animals in the field were followed from nest to nest, for several hours and/or days. All faecal samples were collected immediately after defecation from identified known individuals in conformity with the ethical treatment of non-human primates. Collected samples were preserved in ethanol (96\%). Given codes, sex, animal category, population and location were recorded for each animal (Supp Table 1). 
Collection of eggs and microscopy. For each sample; two grams of faecal material was homogenised with $15 \mathrm{ml}$ water and filtered thought a $100 \mu \mathrm{m}$ sieve $(\sim)$ for microscopic examination. The filtered material was then examined using Sheather's flotation solution ${ }^{63}$ and the sedimentation method, recommended in the approved guidelines of the Clinical and Laboratory Standards Institute for identification of intestinal tract parasites $^{64}$. The whole sediment sample examination was repeated several times after the same sedimentation. All observed eggs were examined under an Olympus BX50 light microscope under the magnification X100. Egg size, shape, colour and internal structures were recorded. Eggs tentatively identified as Oesophagostomum spp., based on morphological characteristics, were transferred using a micropipette to eppendorf tubes contain $200 \mu \mathrm{l}$ PBS for molecular confirmation.

DNA extraction, PCR conditions and sequencing. The isolated eggs from one individual were combined into one tube and were washed and centrifuged twice with $200 \mu \mathrm{l}$ PBS to remove any residual ethanol. DNA was extracted using the QIAamp DNA mini kit. DNA was purified from tissues (Qiagen, France) according to the manufacturer's recommendation with the following modifications: samples were subsequently mixed with $0.2 \mathrm{~g}$ sterile mixture of $0.1 / 0.5 \mathrm{~mm}$ glass beads (Bertin Technologies, www.precellys.com) and $180 \mu \mathrm{l}$ of ATL buffer (Qiagen). Samples were homogenised using a TissueLyser (Qiagen Retsch GmbH, Hannover, Germany) for $5 \mathrm{~min}$ at maximum speed. Hereafter, the suspensions were incubated over night at $56^{\circ} \mathrm{C}$. DNA was eluted in $70 \mu \mathrm{l}$ of distillate water.

The entire ITS1-5.8S-ITS2 region of the Oesophagostomum genus was amplified by a two-step semi nested PCR using forward and reverse primers (Supp Table 2) flanking the $3^{\prime}$ terminus of $18 \mathrm{~S}$ rDNA and the $5^{\prime}$ terminus of $28 \mathrm{~S} r$ DNA, respectively. PCR amplification was performed in $25 \mu$ containing $12.5 \mu \mathrm{l}$ AmpliTaq Gold Master Mix, $0.5 \mu \mathrm{l}$ of $10 \mu \mathrm{M}$ each primer and $5 \mu \mathrm{l}$ of template. The second of the two nested PCR steps was done following the same protocol using $3 \mu \mathrm{l}$ of the first PCR product as template. For each run, as a negative control, nuclease-free water was added to the PCR mix instead of the DNA sample.

The PCR I cycles were: $95^{\circ} \mathrm{C}(10 \mathrm{~min}),\left[95^{\circ} \mathrm{C}(30 \mathrm{~s}), 55^{\circ} \mathrm{C}(30 \mathrm{~s}), 72^{\circ} \mathrm{C}(1 \mathrm{~min})\right]$ for $40 \mathrm{cycles}$ and $7 \mathrm{~min}$ at $72^{\circ} \mathrm{C}$. The PCR IIa cycles were: $95^{\circ} \mathrm{C}(10 \mathrm{~min}),\left[95^{\circ} \mathrm{C}(30 \mathrm{~s}), 53^{\circ} \mathrm{C}(30 \mathrm{~s}), 72^{\circ} \mathrm{C}(45 \mathrm{~s})\right]$ for 40 cycles and $7 \mathrm{~min}$ at $72^{\circ} \mathrm{C}$. The PCR IIb: $95^{\circ} \mathrm{C}(10 \mathrm{~min}),\left[95^{\circ} \mathrm{C}(30 \mathrm{~s}), 55^{\circ} \mathrm{C}(30 \mathrm{~s}), 72^{\circ} \mathrm{C}(45 \mathrm{~s})\right]$ for 40 cycles and $7 \mathrm{~min}$ at $72{ }^{\circ} \mathrm{C}$.

The amplified products were subjected to $2 \%$ agarose gel electrophoresis in TAE buffer. PCR products were commercially purified and sequenced in both directions using nested PCR primers by Macrogen (Macrogen, Amsterdam, Netherlands).

Statistical analysis. Only PCR positive samples were taken for analysis. Statistical analysis was carried out using $\mathrm{R}$ version $3.5 .1^{65}$. Generalized linear models are recommended for the analyses of parasite data but as full factorial or simpler models could not be fitted due to lack of convergence, $\chi 2$ tests were used. Tests carried out were two-tailed with Yates correction. Animals were deemed positive if any faecal sample collected from them during the study tested positive by PCR. A p $\leq 0.05$ was considered statistically significant. For more details, see Stuart et $\mathrm{al}^{12}$.

Sequence alignment, sequences and phylogenetic analyses. All sequences were manually checked, assembled and concatenated using CodonCode Aligner software (www.codoncode.com). Sequences were aligned using Clustal W with Bioedit software version 7.2.5.

For each population, aligned FASTA files were collapsed into variable sites and haplotypes for parsimony network were reconstructed using DNASP v.5.10.0166. A statistical parsimony network to infer relationships among sequences was created with Network $5.0^{67}$.

To examine the relationship of the all known Oesophagostomum species to date, a phylogenetic tree was constructed using a set of reference sequences belonging to different species from Genbank (Supplementary Table S4). For a more descriptive tree, only two of the multiple representative sequences of a haplotype were used in analysis. Phylogenetic analyses were then performed after multiple alignments of the obtained partial ITS1-5.8S-ITS2 sequences (258 nucleotides). We used both Bayesian (Fig. 3) and maximum likelihood (ML) (Supplementary Figure S2) methods for phylogenetic tree construction. The best-fitting ML model under the Akaike information criterion was HKY86 + G6 for nucleotides as identified by JModelTest v.0.1.1 ${ }^{40}$.

For phylogenetic Bayesian inferences, the software MrBayes 3.2.6_ $1^{68}$ was performed using the web service of Phylogeny.fr (https://ngphylogeny.fr/tools/tool/281/form). Markov Chain Monte Carlo (MCMC) parameters were set to sample a tree every 10 or 100 generations of 100.000 generations and the burn-in was set at 250 trees sampled. Resulted tree was accepted when the average standard deviation of split frequencies is lower than 0.05 and the average Potential Scale Reduction Factor (PSRF) for parameter values is around 1.0 (0.9-1.1), simultaneously.

The highest-likelihood DNA trees and corresponding bootstrap support values were obtained by PhyML ${ }^{69}$ (freely available at the ATGC bioinformatics platform http://www.atgc-montpellier.fr/) using nearest neighbour interchange plus subtree pruning recrafting (NNI + SPR) branch swapping and 100 bootstrap replicates. Necator americanus (LC036563) was used to root the tree. The resulting tree was drawn using iTOL v.5.4 ${ }^{70}$.

Genetic diversity and population differentiation. The genetic diversity estimates (N, Number of sequence obtained; S, Number of polymorphic sites; K, Number of haplotypes; Hd, Haplotype diversity; $\pi$, Nucleotide diversity; D, Average number of nucleotide difference between populations) were computed using DNASP v.5.10.01 ${ }^{66}$. Finally, to evaluate whether the genetic differentiation between populations was associated with the geographical isolation of islands, analysis of molecular variance AMOVA was performed in Arlequin 
ver 3.1 $1^{71}$. Mantel test for matrix correlation between genetic distance and geographic distance was performed by using IBD ${ }^{72}$ with 1000 permutations.

Ethics approval. All the research reported in this manuscript adhered to the legal requirements of the country in which the work took place. Since the collection of faecal samples from orangutans and gibbons was non-invasive and did not involve interaction with or distress to the animals, the study was not reviewed by an animal ethics committee.

\section{Data avaliability}

The newly generated sequences were deposited in the GenBank database under the accession numbers: MW756966-MW756992.

Received: 27 October 2020; Accepted: 16 March 2021

Published online: 31 March 2021

\section{References}

1. Locke, D. P. et al. Comparative and demographic analysis of orang-utan genomes. Nature 469, 529-533. https://doi.org/10.1038/ nature09687 (2011).

2. Nater, A. et al. Morphometric, behavioral, and genomic evidence for a new orangutan species. Curr. Biol. 27, 3487-3498 e3410. https://doi.org/10.1016/j.cub.2017.09.047 (2017).

3. Kim, S. K. et al. Patterns of genetic variation within and between Gibbon species. Mol. Biol. Evol. 28, 2211-2218 (2011).

4. Thinh, V. N. et al. Mitochondrial evidence for multiple radiations in the evolutionary history of small apes. BMC Evol. Biol. 10, 74. https://doi.org/10.1186/1471-2148-10-74 (2010).

5. IUCN. IUCN Red List of Threatened Species. http://www.redlist.org (2019).

6. Kilbourn, A. M. et al. Health evaluation of free-ranging and semi-captive orangutans (Pongo pygmaeus pygmaeus) in Sabah, Malaysia. J. Wildl. Dis. 39, 73-83. https://doi.org/10.7589/0090-3558-39.1.73 (2003).

7. Klaus, A. et al. Co-infection patterns of intestinal parasites in arboreal primates (proboscis monkeys, Nasalis larvatus) in Borneo. Int J Parasitol Parasites Wildl 6, 320-329. https://doi.org/10.1016/j.ijppaw.2017.09.005 (2017).

8. Labes, E. M. et al. Intestinal parasites of endangered orangutans (Pongo pygmaeus) in Central and East Kalimantan, Borneo, Indonesia. Parasitology 137, 123-135. https://doi.org/10.1017/S0031182009991120 (2010).

9. Goldberg, T. L. et al. Fatal metacestode infection in Bornean orangutan caused by unknown Versteria species. Emerg. Infect Dis. 20, 109-113. https://doi.org/10.3201/eid2001.131191 (2014).

10. Ihms, E. A., Daniels, J. B., Koivisto, C. S., Barrie, M. T. \& Russell, D. S. Fatal Streptococcus anginosus-associated pneumonia in a captive Sumatran orangutan (Pongo abelii). J. Med. Primatol. 43, 48-51. https://doi.org/10.1111/jmp.12085 (2014).

11. Nidom, C. A. et al. Serological evidence of Ebola virus infection in Indonesian orangutans. PLoS ONE 7, e40740. https://doi.org/ 10.1371/journal.pone.0040740 (2012).

12. Stuart, P. et al. Entamoeba histolytica infections in wild and semi-wild orangutans in Sumatra and Kalimantan. Am. J. Primatol. 82, e23124. https://doi.org/10.1002/ajp.23124 (2020).

13. Utsumi, T. et al. Full genome characterization and phylogenetic analysis of hepatitis B virus in gibbons and a caretaker in Central Kalimantan, Indonesia. Arch Virol. 160, 685-692 (2015).

14. Collet, J. Y., Galdikas, B. M. F., Sugarjito, J. \& Jojosudharmo, S. A coprological study of parasitism in orangutans (Pongo-Pygmaeus) in Indonesia. J. Med. Primatol. 15, 121-129 (1986).

15. Foitova, I., Civanova, K., Barus, V. \& Nurcahyo, W. Phylogenetic relationships between pinworms (Nematoda: Enterobiinae) parasitising the critically endangered orang-utan, according to the characterisation of molecular genomic and mitochondrial markers. Parasitol. Res. 113, 2455-2466. https://doi.org/10.1007/s00436-014-3892-y (2014).

16. Foitova, I. et al. Redescription and resurrection of Bertiella satyri (Cestoda, Anoplocephalidae) parasitizing the orangutan (Pongo abelii) in Indonesia. Parasitol. Res. 109, 689-697. https://doi.org/10.1007/s00436-011-2300-0 (2011).

17. Pacheco, M. A. et al. The origin of malarial parasites in orangutans. PLoS ONE 7, e34990. https://doi.org/10.1371/journal.pone. 0034990 (2012).

18. Xie, Y. et al. Complete mitochondrial genomes of chimpanzee- and gibbon-derived Ascaris isolated from a zoological garden in southwest China. PLoS ONE 8, e82795 (2013).

19. Hasegawa, H. et al. Humans and great apes cohabiting the forest ecosystem in central african republic harbour the same hookworms. PLoS Negl Trop Dis 8, e2715. https://doi.org/10.1371/journal.pntd.0002715 (2014).

20. Huffman, M. A., Gotoh, S., Turner, L. A., Hamai, M. \& Yoshida, K. Seasonal trends in intestinal nematode infection and medicinal plant use among chimpanzees in the Mahale Mountains, Tanzania. . Primates 38 (1997).

21. Kalousova, B. et al. Diversity and transmission of soil transmitted strongylid nematodes between humans and other primates in the wild. J. Nematol. 46, 182-182 (2014).

22. Nejsum, P., Bertelsen, M. F., Betson, M., Stothard, J. R. \& Murrell, K. D. Molecular evidence for sustained transmission of zoonotic Ascaris suum among zoo chimpanzees (Pan troglodytes). Vet. Parasitol. 171, 273-276 (2010).

23. Ravasi, D. F., O’Riain, M. J., Davids, F. \& Illing, N. Phylogenetic Evidence That Two Distinct Trichuris Genotypes Infect both Humans and Non-Human Primates. Plos One 7 (2012).

24. Seguel, M. \& Gottdenker, N. The diversity and impact of hookworm infections in wildlife. Int. J. Parasitol. Par. 6, 177-194 (2017).

25. Thanchomnang, T. et al. First molecular identification and genetic diversity of Strongyloides stercoralis and Strongyloides fuelleborni in human communities having contact with long-tailed macaques in Thailand. Parasitol. Res. 116, 1917-1923 (2017).

26. Huffman, M. A. \& Caton, J. M. Self-induced increase of gut motility and the control of parasitic infections in wild chimpanzees. Int. J. Primatol. 22, 329-346 (2001).

27. Makouloutou, P. et al. Prevalence and genetic diversity of Oesophagostomum stephanostomum in wild lowland gorillas at Moukalaba-Doudou National Park, Gabon. Helminthologia 51, 83-93. https://doi.org/10.2478/s11687-014-0214-y (2014).

28. Ghai, R. R., Chapman, C. A., Omeja, P. A., Davies, T. J. \& Goldberg, T. L. Nodule worm infection in humans and wild primates in Uganda: cryptic species in a newly identified region of human transmission. PLoS Negl. Trop. Dis. 8, e2641. https://doi.org/10. 1371/journal.pntd.0002641 (2014).

29. Patrelle, C., Ferte, H. \& Jouet, D. Identification of Chabertiidae (Nematoda, Strongylida) by PCR-RFLP based method: a new diagnostic tool for cross transmission investigation between domestic and wild ruminants in France. Infect. Genet. Evol. 28, 15-20 (2014).

30. Petersen, H. H., Andreasen, A., Kringel, H., Roepstorff, A. \& Thamsborg, S. M. Parasite population dynamics in pigs infected with Trichuris suis and Oesophagostomum dentatum. Vet Parasitol 199, 73-80 (2014). 
31. Schar, F. et al. The prevalence and diversity of intestinal parasitic infections in humans and domestic animals in a rural Cambodian village. Parasitol. Int. 63, 597-603 (2014).

32. Blotkamp, J. et al. Observations on the morphology of adults and larval stages of Oesophagostomum sp. isolated from man in northern Togo and Ghana. J Helminthol 67, 49-61 (1993).

33. Polderman, A. M. \& Blotkamp, J. Oesophagostomum infections in humans. Parasitol Today 11, 451-456 (1995).

34. Polderman, A. M., Krepel, H. P., Baeta, S., Blotkamp, J. \& Gigase, P. Oesophagostomiasis, a common infection of man in northern Togo and Ghana. Am. J. Trop. Med. Hyg. 44, 336-344 (1991).

35. Krief, S. et al. Clinical and pathologic manifestation of oesophagostomosis in African great apes: does self-medication in wild apes influence disease progression?. J Med Primatol 37, 188-195 (2008).

36. Nurcahyo, W., Konstanzova, V. \& Foitova, I. Parasites of orangutans (primates: ponginae): an overview. Am J Primatol 79. https:// doi.org/10.1002/ajp.22650 (2017).

37. Arizono, N., Yamada, M., Tegoshi, T. \& Onishi, K. Molecular Identification of Oesophagostomum and Trichuris Eggs Isolated from Wild Japanese Macaques. Korean J. Parasitol. 50, 253-257. https://doi.org/10.3347/kjp.2012.50.3.253 (2012).

38. Frias, L. et al. Molecular characterization of nodule worm in a community of Bornean primates. Ecol Evol 9, 3937-3945. https:// doi.org/10.1002/ece3.5022 (2019).

39. Gillespie, T. R., Greiner, E. C. \& Chapman, C. A. Gastrointestinal parasites of the colobus monkeys of Uganda. J Parasitol 91, 569-573. https://doi.org/10.1645/GE-434R (2005).

40. Posada, D. jModelTest: phylogenetic model averaging. Mol Biol Evol 25, 1253-1256. https://doi.org/10.1093/molbev/msn083 (2008).

41. Blouin, M. S. Molecular prospecting for cryptic species of nematodes: mitochondrial DNA versus internal transcribed spacer. Int J Parasitol 32, 527-531. https://doi.org/10.1016/s0020-7519(01)00357-5 (2002).

42. Pafco, B. et al. Metabarcoding analysis of strongylid nematode diversity in two sympatric primate species. Sci Rep 8, 5933. https:// doi.org/10.1038/s41598-018-24126-3 (2018).

43. De Gruijter, J. M., Ziem, J., Verweij, J. J., Polderman, A. M. \& Gasser, R. B. Genetic substructuring within Oesophagostomum bifurcum (Nematoda) from human and non-human primates from Ghana based on random amplified polymorphic DNA analysis. Am. J. Trop. Med. Hyg 71, 227-233 (2004).

44. Gasser, R. B., de Gruijter, J. M. \& Polderman, A. M. Insights into the epidemiology and genetic make-up of Oesophagostomum bifurcum from human and non-human primates using molecular tools. Parasitology 132, 453-460 (2006).

45. Krief, S. et al. Nodular worm infection in wild chimpanzees in Western Uganda: a risk for human health?. PLoS Negl. Trop. Dis. 4, e630. https://doi.org/10.1371/journal.pntd.0000630 (2010).

46. Ota, N. et al. Molecular identification of Oesophagostomum spp. from 'village' chimpanzees in Uganda and their phylogenetic relationship with those of other primates. $R$ Soc Open Sci 2, 150471 (2015).

47. Mul, I. F., Paembonan, W., Singleton, I., Wich, S. A. \& van Bolhuis, H. G. Intestinal parasites of free-ranging, semicaptive, and captive Pongo abelii in sumatra, indonesia. Int J Primatol 28, 407-420. https://doi.org/10.1007/s10764-007-9119-7 (2007).

48. Mynarova, A. et al. Prevalence of Cryptosporidium spp., Enterocytozoon bieneusi, Encephalitozoon spp. and Giardia intestinalis in Wild, Semi-Wild and Captive Orangutans (Pongo abelii and Pongo pygmaeus) on Sumatra and Borneo, Indonesia. Plos One 11, e0152771. https://doi.org/10.1371/journal.pone.0152771 (2016).

49. Labes, E. M., Nurcahyo, W., Deplazes, P. \& Mathis, A. Genetic characterization of Strongyloides spp. from captive, semi-captive and wild Bornean orangutans (Pongo pygmaeus) in Central and East Kalimantan, Borneo, Indonesia. Parasitology 138, 1417-1422. https://doi.org/10.1017/S0031182011001284 (2011).

50. Reid, M. J. et al. Transmission of human and macaque Plasmodium spp. to ex-captive orangutans in Kalimantan, Indonesia. Emerg Infect Dis. 12, 1902-1908 (2006).

51. Vitone, N. D., Altizer, S. \& Nunn, C. L. Body size, diet and sociality influence the species richness of parasitic worms in anthropoid primates. Evol. Ecol. Res. 6, 183-199 (2004).

52. Campbell-Smith, G., Campbell-Smith, M., Singleton, I. \& Linkie, M. Apes in space: saving an imperilled orangutan population in Sumatra. PLoS ONE 6, e17210 (2011).

53. Huffman, M. A. Self-medicative behavior in the African great apes: an evolutionary perspective into the origins of human traditional medicine. Bioscience 51, 651-661 (2001).

54. Huffman, M. A. et al. Leaf-swallowing by chimpanzees: a behavioral adaptation for the control of strongyle nematode infections. Int J Primatol 17, 475-503 (1996).

55. Barelli, C. \& Huffman, M. A. Leaf swallowing and parasite expulsion in Khao Yai white-handed gibbons (Hylobates lar), the first report in an Asian ape species. Am J Primatol 79, 1-7. https://doi.org/10.1002/ajp.22610 (2017).

56. Dubey, J. P. et al. Characterization of Toxoplasma gondii from raccoons (Procyon lotor), coyotes (Canis latrans), and striped skunks (Mephitis mephitis) in Wisconsin identified several atypical genotypes. J Parasitol 93, 1524-1527 (2007).

57. Mercier, A. et al. Human impact on genetic diversity of Toxoplasma gondii: example of the anthropized environment from French Guiana. Infect. Genet. Evol. 11, 1378-1387 (2010).

58. Phuphisut, O. et al. Molecular identification of the strongyloid nematode Oesophagostomum aculeatum in the Asian wild elephant Elephas maximus. J Helminthol, 1-7 (2015).

59. Malaivijitnond, S., Chaiyabutr, N., Urasopon, N. \& Hamada, Y. in Proceedings of the 32nd Congress on Science and Technology of Thailand. 73-77.

60. Kamel, A. G. M., Yang, C. O. \& Norazah, A. Oesophagostomum aculeatum, the nodular worm causing helminthoma in man in Malaysia. Int. Med. J. 2, 287-290 (1995).

61. Karim, N. \& Yang, C. O. Oesophagostomiasis in man: report of the first Malaysian case with emphasis on its pathology. Malays. J. Pathol. 14, 19-24 (1992).

62. Ross, R. A., Gibson, D. I. \& Harris, E. A. Cutaneous oesophagostomiasis in man. J Helminthol 63, 261-265 (1989).

63. Sheater, A. L. The detection of intestinal protozoa and mange parasites by a flotation technique. J Comp Pathol 36, 266-275 (1923).

64. Garcia, L. S. et al. Practical Guidance for Clinical Microbiology Laboratories: Laboratory Diagnosis of Parasites from the Gastrointestinal Tract. Clin Microbiol Rev 31. https://doi.org/10.1128/CMR.00025-17 (2018).

65. R: A language and environment for statistical computing. (2018).

66. Rozas, J., Sanchez-DelBarrio, J. C., Messeguer, X. \& Rozas, R. DnaSP, DNA polymorphism analyses by the coalescent and other methods. Bioinformatics 19, 2496-2497 (2003).

67. Bandelt, H. J., Forster, P. \& Rohl, A. Median-joining networks for inferring intraspecific phylogenies. Mol Biol Evol 16, 37-48 (1999).

68. Huelsenbeck, J. P. \& Ronquist, F. MRBAYES: Bayesian inference of phylogenetic trees. Bioinformatics 17, 754-755. https://doi.org/ 10.1093/bioinformatics/17.8.754 (2001).

69. Guindon, S. et al. New algorithms and methods to estimate maximum-likelihood phylogenies: assessing the performance of PhyML 3.0. Syst Biol 59, 307-321. https://doi.org/10.1093/sysbio/syq010 (2010).

70. Letunic, I. \& Bork, P. Interactive Tree Of Life (iTOL) v4: recent updates and new developments. Nucleic Acids Res 47, W256-W259. https://doi.org/10.1093/nar/gkz239 (2019).

71. Excoffier, L., Laval, G. \& Schneider, S. Arlequin (version 3.0): an integrated software package for population genetics data analysis. Evol Bioinform Online 1, 47-50 (2005). 
72. Bohonak, A. J. IBD (Isolation by Distance): a program for analyses of isolation by distance. J Hered 93, 153-154. https://doi.org/ 10.1093/jhered/93.2.153 (2002).

73. Husson, S. J. et al. Orangutans geographic variation in behavioral ecology and conservation. In S. A. Wich, S. U. Atmoko, T. M. Setia \& C. P. van Schaik (Eds.), Orangutan distribution, density, abundance and impacts of disturbance. 77-96 (2009).

74. Cheyne, S. M., Thompson, C. J., Phillips, A. C., Hill, R. M. \& Limin, S. H. Density and population estimate of gibbons (Hylobates albibarbis) in the Sabangau catchment, Central Kalimantan. Indonesia. Primates 49, 50-56. https://doi.org/10.1007/s10329-0070063-0 (2008).

75. van Schaik, C. P. The socioecology of fission-fusion sociality in Orangutans. Primates 40, 69-86. https://doi.org/10.1007/BF025 57703 (1999).

\section{Acknowledgements}

Authors would like to thank the Indonesian Institute of Sciences (LIPI), the State Ministry of Research and Technology (RISTEK) and Directorate General for Natural Conservation (PHKA) for granting permission to conduct research in Indonesia (permits number-3576/SU/KS/2004, 00217/SU/KS/2005, 04208/SU/KS/2006, 2320/SU/ KS/2007, 0056/FRP/SM/III/2009, 0008/EXT/FRP/SM/II/2010, CITES permit 06968/IV/SATS-LN/, CITES permit 16074/IV/SATS-LN/2008, CITES permit08411/IV/SATS-LN/2011). The authors would like to thanks to Šárka Mašová for map preparation. The authors also would like to thank to Carel van Schaik for hosting us in Suaq. We also thank to Andrea Permana for help in the field. The study was financially supported by the Foundation "UMI-Saving of Pongidae" with partial support by Czech Academy of Sciences Grants P505/11/1163. This work was supported by the Program of Employment of Newly Graduated Doctors of Science for Scientific Excellence" (grant number CZ.1.07/2.3.00/30.0009) and (grant number CZ.1.07/2.3.00/30.0037), co-financed from European Social Fund and the state budget of the Czech Republic. The work done in the lab of Adrian Streit was funded by the Max-Planck-Society.

\section{Author contributions}

E.Y. and I.F. designed research; E.Y., P.S., H.H., A.S. and J.D. performed research; I.F. coordinated field work, sample, data collection and supervised the project; H.M.B., S.M.C., W.N. contributed to the field study design and field coordination; E.Y. drafted the first version of manuscript; P.S., H.H., A.S., J.D., H.M.B., S.M.C. W.N. and I.F. have been involved in revising the manuscript. All authors read and approved the final manuscript.

\section{Competing interests}

The authors declare no competing interests.

\section{Additional information}

Supplementary Information The online version contains supplementary material available at https://doi.org/ 10.1038/s41598-021-86518-2.

Correspondence and requests for materials should be addressed to I.F.

Reprints and permissions information is available at www.nature.com/reprints.

Publisher's note Springer Nature remains neutral with regard to jurisdictional claims in published maps and institutional affiliations.

(c) (i) Open Access This article is licensed under a Creative Commons Attribution 4.0 International License, which permits use, sharing, adaptation, distribution and reproduction in any medium or format, as long as you give appropriate credit to the original author(s) and the source, provide a link to the Creative Commons licence, and indicate if changes were made. The images or other third party material in this article are included in the article's Creative Commons licence, unless indicated otherwise in a credit line to the material. If material is not included in the article's Creative Commons licence and your intended use is not permitted by statutory regulation or exceeds the permitted use, you will need to obtain permission directly from the copyright holder. To view a copy of this licence, visit http://creativecommons.org/licenses/by/4.0/.

(C) The Author(s) 2021 